\title{
THE DFR PROPERTY FOR COUNTING PROCESSES STOPPED AT AN INDEPENDENT RANDOM TIME
}

\author{
F. G. BADíA* AND \\ C. SANGÜESA, ${ }^{* *}$ University of Zaragoza
}

\begin{abstract}
In this paper we consider general counting processes stopped at a random time $T$, independent of the process. Provided that $T$ has the decreasing failure rate (DFR) property, we present sufficient conditions on the arrival times so that the number of events occurring before $T$ preserves the DFR property of $T$. In particular, when the interarrival times are independent, we consider applications concerning the DFR property of the stationary number of customers waiting in queue for specific queueing models.
\end{abstract}

Keywords: Decreasing failure rate; counting process; renewal process; stochastic order; association; Little's law

2010 Mathematics Subject Classification: Primary 62E10

Secondary 60E15

\section{Introduction}

The Decreasing failure rate (DFR) is a property observed in systems which improve with age. Although counterintuitive, this property appears in real life. One of the first examples was observed by Proschan [16] in the study of failures of air-conditioning systems in aeroplanes. The theoretical explanation of these observed DFRs is that the data can be described by a mixture model of exponential distributions. Although exponential distributions have a constant failure rate, their mixtures are DFR. In fact, the preservation of the DFR property under mixing is well-known. In addition, mixtures of increasing failure rate (IFR) distributions may show DFRs; see, for example, [11] and [9].

The aim of this paper is to show that counting processes stopped at a DFR random time inherit this property under rather general assumptions on the interarrival times. To the best of the authors ' knowledge, one of the first publications regarding the preservation of properties in counting processes observed at a random time (classical renewal processes) was Esary et al. [7]. The special case of the Poisson process has been the subject of much attention (cf. Grandell [10, Chapter 7] and the references therein). Ross et al. [17, Theorem 3.1] studied the preservation of the IFR property in general renewal processes. This subject was also studied in [2], together with the preservation of the decreasing reversed hazard rate property.

Several examples of practical applications of counting processes stopped at a random time are described in [6, p. 42]. For example, $T$ may be the lifetime of a whole piece of equipment, and we may be concerned with the number of component replacements, $N(T)$, that are necessary during $T$. In several queueing models, the stationary length of the queue can be modeled by means of a random variable (RV) $N(T)$ (see [1] and [12]), with $T$ being the stationary waiting

Received 3 November 2011; revision received 12 May 2014.

* Postal address: Maria de Luna 3, Zaragoza, 50018, Spain. Email address: gbadia@ unizar.es

** Postal address: Pedro Cerbuna 11, Zaragoza, 50009, Spain. Email address: csangues@ unizar.es 
time and $N$ being the equilibrium renewal processes of the arrival of customers in the queue. Recent applications have been found in the health sciences (see [20]), in which the counting process describes the number of tests for a disease (HIV for example) in a person at risk of infection until the random time $T$ at which this person is infected. Several more applications are described in [3] and the references therein.

The starting point of this paper is [4], in which it was shown that in a classical renewal process (independent and identically distributed (i.i.d.) interarrival times) the DFR (as well as the logconvexity) is preserved. In Theorem 3.1 we extend this property to possibly dependent interarrival times. As a particular case, we consider independent and stochastically decreasing interarrival times (see Corollary 3.1) and apply it to queueing models (see Section 4). More specifically, we deduce the DFR property of the stationary number of customers waiting in queue for specific queueing models in Corollaries 4.2 and 4.3. Finally, in Section 5 we present two examples of counting processes with dependent interarrival times (see Corollaries 5.1 and 5.2) which satisfy the conditions in Theorem 3.1.

In the next section we introduce the main concepts which we are going to use throughout the paper.

\section{Preliminaries}

We consider a general counting process $\{N(t): t \geq 0\}$ defined as follows. The arrival times in the counting process $\{N(t): t \geq 0\}$ are denoted by $S_{n}, n=0,1, \ldots$ where $S_{0}=0$, and the interarrival times are denoted by $\left(X_{n}\right)_{n=1,2, \ldots}$. The arrival and interarrival times satisfy the identity

$$
S_{n}=\sum_{i=1}^{n} X_{i}, \quad n=1,2, \ldots
$$

The only assumption concerning the $X_{n}$ is that they are nonnegative RVs and are not degenerate at 0 . The general counting process is defined through the renewal epochs by the following expression:

$$
N(t)=\max \left\{n: S_{n} \leq t\right\}, \quad t \geq 0 .
$$

This paper deals with the preservation of the DFR property. Firstly, we recall below the definition of this reliability class (see [5]), along with some other definitions of reliability classes that are weaker than the DFR property, which will be used throughout the paper. The terms 'increasing' and 'decreasing' mean, as usual, 'nondecreasing' and 'nonincreasing', respectively.

Definition 2.1. Let $X$ be a nonnegative RV with $G$ and $\bar{G}:=1-G$ the corresponding distribution and reliability functions. Then $X$ (or $G$ ) is said to be:

(i) DFR if $\bar{G}(z+t) / \bar{G}(t)$ is increasing in $t$ for all $z \geq 0$,

(ii) new worse than used (NWU) if $\bar{G}(z) \bar{G}(t) \leq \bar{G}(z+t)$, for all $z, t \geq 0$,

(iii) new worse than used in expectation (NWUE) if $\mu:=\mathbb{E}\{X\} \leq \int_{0}^{\infty} \bar{G}(z+t) / \bar{G}(t) \mathrm{d} z$ for all $t \geq 0$,

(iv) increasing mean residual life (IMRL) if $m(t):=\int_{0}^{\infty} \bar{G}(z+t) / \bar{G}(t) \mathrm{d} z$ is increasing in $t \geq 0$. 
Note that if the monotonicity and sense of the inequalities in Definition 2.1 are reversed, we obtain the dual concepts of IFR, new better than used, new better than used in expectation, and decreasing mean residual life.

Remark 2.1. We list some properties of the previous definitions which will be used throughout the paper.

(i) A DFR distribution can show a jump only at the origin, and the interval of support of DFR distributions is $[0, \infty)$ (see [14, p. 117])).

(ii) If $\bar{G}$ is a DFR survival function, and we take $t \geq 0$ fixed then the function defined by $\bar{H}(z)=\bar{G}(z+t) / \bar{G}(t), z \geq 0$ (residual life survival function) is also a DFR survival function (see [14, p. 118]).

(iii) DFR implies NWU, NWUE, and IMRL (see [14, p. 181]).

To describe the DFR property for counting processes, we will use the definition of this property for discrete RVs (see, for example, [7] or [10, Chapter 7]), which is recalled in the following definition.

Definition 2.2. Let $X$ be a (possibly defective) nonnegative integer-valued random variable and $p_{n}$ its corresponding probability mass function

$$
p_{n}:=\mathbb{P}\{X=n\} \quad \text { for } n=0,1, \ldots,
$$

where $\mathbb{P}$ is the probability measure. The distribution function is said to be discrete decreasing failure rate (dDFR) if $\mathbb{P}\{X=n\} / \mathbb{P}\{X \geq n\}$ is decreasing in $n$ or, equivalently, if

$$
\mathbb{P}\{X \geq n+1\}^{2} \leq \mathbb{P}\{X \geq n\} \mathbb{P}\{X \geq n+2\} \text { for } n=0,1, \ldots
$$

Some of the conditions in our results require the comparison of RVs with respect to the usual stochastic order, which we recall in the following definitions and remarks.

Definition 2.3. Let $X$ and $Y$ be two RVs with distribution functions $F_{X}$ and $F_{Y}$, respectively. The RV $X$ is said to be smaller than $Y$ in the usual stochastic order (denoted by $X \leq \mathrm{sT} Y$ ) if $F_{X}(t) \geq F_{Y}(t)$ for all real $t$ or, equivalently, if $\bar{F}_{X}(t) \leq \bar{F}_{Y}(t)$ for all real $t$.

We will write $X={ }_{\mathrm{ST}} Y$ to indicate that the RVs $X$ and $Y$ have the same distribution function.

Remark 2.2. The following property of the usual stochastic order can be found in $[15, \mathrm{p} .6]$, for example. Let $X$ and $Y$ be two RVs. $X \leq_{\text {ST }} Y$ if and only if $\mathbb{E}\{h(X)\} \leq(\geq) \mathbb{E}\{h(Y)\}$ for all increasing (decreasing) functions $h$ such that the expectations exist.

The next concept of association between RVs, introduced in Esary et al. [8], will also play an important role in our results. In what follows, given two vectors $\boldsymbol{x}:=\left(x_{1}, \ldots x_{n}\right) \in \boldsymbol{R}^{n}$ and $\boldsymbol{y}:=\left(y_{1}, \ldots, y_{n}\right) \in \boldsymbol{R}^{n}$, the notation $\boldsymbol{x} \leq \boldsymbol{y}$ will be used to mean $x_{i} \leq y_{i}, i=1,2, \ldots, n$. A function $f: \boldsymbol{R}^{n} \rightarrow \boldsymbol{R}$ will be said to be increasing if $\boldsymbol{x} \leq \boldsymbol{y}$ implies that $f(\boldsymbol{x}) \leq f(\boldsymbol{y})$ (which is the same thing as saying that $f$ is componentwise increasing).

Definition 2.4. A random vector $\boldsymbol{X}:=\left(X_{1}, \ldots, X_{n}\right)$ is said to be associated if

$$
\operatorname{cov}(f(\boldsymbol{X}), g(\boldsymbol{X}))=\mathbb{E}\{f(\boldsymbol{X}) g(\boldsymbol{X})\}-\mathbb{E}\{f(\boldsymbol{X})\} \mathbb{E}\{g(\boldsymbol{X})\} \geq 0
$$

for all increasing functions $f, g: \boldsymbol{R}^{n} \rightarrow \boldsymbol{R}$ such that the expectation exists. 
Remark 2.3. It will be useful throughout the paper to point out that for an associated vector, (2.1) also holds whenever $f$ and $g$ are decreasing. One only has to apply the definition to the increasing functions $-f$ and $-g$.

Remark 2.4. The following interesting properties of association can be found in [14, p. 681] and [15, p. 123], for example.

(i) If $\boldsymbol{X}$ is associated and $f_{i}: \boldsymbol{R}^{n} \rightarrow \boldsymbol{R}, i=1,2, \ldots, m, m \in \mathbb{N}$ are all increasing (or all decreasing), then $\left(f_{1}(\boldsymbol{X}), \ldots, f_{m}(\boldsymbol{X})\right)$ is associated.

(ii) If $\boldsymbol{X}:=\left(X_{1}, \ldots, X_{n}\right)$ has independent components then it is associated.

(iii) The association property is closed under weak convergence. That is, if we have a sequence of associated random vectors $\left(\boldsymbol{X}_{k}\right)_{k=1,2, \ldots}$ converging in distribution to $\boldsymbol{X}$ then $\boldsymbol{X}$ is associated.

Finally, we introduce some notation, concerning conditional distributions, which play an important role in the proofs of our results. Given a nonnegative random vector $(\boldsymbol{X}, \boldsymbol{Y}$, in which $\boldsymbol{X}$ has dimension $n$ and $\boldsymbol{Y}$ has dimension $m$, we will denote by $\left\{F_{\{\boldsymbol{Y} \mid \boldsymbol{x}\}}, \boldsymbol{x} \in \mathbb{R}_{+}^{n}\right\}$ a (regular) conditional distribution of $\boldsymbol{y}$ given $\boldsymbol{X}$, that is, a family of distribution functions satisfying for all Borel sets $A \subseteq \boldsymbol{R}_{+}^{n}, B \subseteq \boldsymbol{R}_{+}^{m}$,

$$
\int_{\{\boldsymbol{x} \in A, \boldsymbol{y} \in B\}} \mathrm{d} F_{(\boldsymbol{X}, \boldsymbol{Y})}(\boldsymbol{x}, \boldsymbol{y})=\int_{\boldsymbol{x} \in A} \mathrm{~d} F_{\boldsymbol{X}}(\boldsymbol{x}) \int_{\boldsymbol{y} \in B} \mathrm{~d} F_{\{\boldsymbol{Y} \mid \boldsymbol{x}\}}(\boldsymbol{y}) .
$$

The existence of such a family of conditional distribution functions is always guaranteed (cf. [13, p.107]). Moreover, if in a counting process we consider $\boldsymbol{Y}=\left(X_{n+1}, \ldots, X_{n+m}\right)$, and take $\boldsymbol{X}$ a random vector of dimension $n$ containing the previous interarrival times, for each $\boldsymbol{x} \in \mathbb{R}_{+}^{n}$, we will denote by $\left(Z_{n+1}^{x}, \ldots, Z_{n+m}^{x}\right)$ a random vector whose distribution function is $F_{\left\{\left(X_{n+1}, \ldots, X_{n+m}\right) \mid x\right\}}$. The family of random vectors $\left\{\left(Z_{n+1}^{x}, \ldots, Z_{n+m}^{x}\right), x \in \mathbb{R}_{+}^{n}\right\}$ will be called a distributional version of $\left(X_{n+1}, \ldots, X_{n+m}\right)$ given $\boldsymbol{X}$. In this way, for every function $f: \mathbb{R}_{+}^{n} \times \mathbb{R}_{+}^{m} \rightarrow \mathbb{R}_{+}$, the expected value of $f(\boldsymbol{X}, \boldsymbol{Y})$ can be calculated, thanks to (2.2), as

$$
\mathbb{E}\{f(\boldsymbol{X}, \boldsymbol{Y})\}=\int_{\mathbb{R}_{+}^{n}} \mathbb{E} f\left(\boldsymbol{x}, Z_{n+1}^{\boldsymbol{x}}, \ldots, Z_{n+m}^{\boldsymbol{x}}\right) \mathrm{d} F_{\boldsymbol{X}}(\boldsymbol{x}) .
$$

\section{The DFR property under association and decreasing conditions of the interarrival times}

In this section we present our main result concerning the DFR preservation property in a counting process, making use of the conditional distributions of the interrenewal epochs. Let $\{N(t): t \geq 0\}$ be a general counting process with interrenewal epochs $\left(X_{n}\right)_{n=1,2, \ldots}$. Let $T$ be a DFR random time independent of the process, whose survival function is denoted by $\bar{F}_{T}$. Our aim is to show that if $T$ is DFR then $N(T)$ is dDFR. For technical reasons, we will assume that for each $n=1,2, \ldots$, the distribution functions of $S_{n}$, the $n$th renewal epoch, and $T$ do not have any common points of discontinuity. Then the following equality is verified (cf. [7, p. 642]):

$$
\mathbb{P}\{N(T) \geq n\}=\mathbb{E}\left\{\bar{F}_{T}\left(S_{n}\right)\right\} \text { for } n=1,2, \ldots
$$

Making use of the previous equality, the dDFR property for $N(T)$ can be proved using the following lemma, which will be used extensively throughout this paper. 
Lemma 3.1. Let $\{N(t): t \geq 0\}$ be a general counting process with interarrival times given by $\left(X_{n}\right)_{n=1,2, \ldots}$. Let $T$ be a random time independent of the process. Assume that

(i) $T$ is a DFR $R V$,

(ii) $\mathbb{P}\{T=0\} \mathbb{P}\left\{X_{1}=0\right\}=0$, that is, $T$ and $X_{1}$ do not have simultaneously positive mass at 0 .

Then $N(T)$ is dDFR if and only if

$$
\begin{gathered}
\mathbb{E}^{2}\left\{\bar{F}_{T}\left(X_{1}\right)\right\} \leq \mathbb{E}\left\{\bar{F}_{T}\left(S_{2}\right)\right\}, \\
\mathbb{E}^{2}\left\{\bar{F}_{T}\left(S_{n+1}\right)\right\} \leq \mathbb{E}\left\{\bar{F}_{T}\left(S_{n}\right)\right\} \mathbb{E}\left\{\bar{F}_{T}\left(S_{n+2}\right)\right\} \quad \text { for } n=1,2, \ldots
\end{gathered}
$$

Proof. Under the previous assumptions, we have to prove that $N(T)$ is $\mathrm{dDFR}$, that is,

$$
\mathbb{P}^{2}\{N(T) \geq n+1\} \leq \mathbb{P}\{N(T) \geq n\} \mathbb{P}\{N(T) \geq n+2\} \quad \text { for } n=0,1,2 \ldots
$$

Firstly, note that as $X_{1} \leq \mathrm{sT} S_{n}$, assumption (ii) implies that

$$
\mathbb{P}\{T=0\} \mathbb{P}\left\{S_{n}=0\right\} \leq \mathbb{P}\{T=0\} \mathbb{P}\left\{X_{1}=0\right\}=0 \text { for } n=1,2, \ldots
$$

This means that for each $n=1,2 \ldots, S_{n}$ and $T$ do not have the origin as a common discontinuity point in their distribution functions. Recalling property (i) in Remark 2.1 and that $T$ is DFR, this implies that $S_{n}$ and $T$ do not have any common discontinuity points in their distribution functions, and hence (3.1) holds. Thus, taking into account that $\mathbb{P}\{N(T) \geq 0\}=1$, then (3.4) for $n=0$ becomes

$$
\mathbb{E}^{2}\left\{\bar{F}_{T}\left(X_{1}\right)\right\} \leq \mathbb{E}\left\{\bar{F}_{T}\left(S_{2}\right)\right\},
$$

whereas for $n=1,2, \ldots$, (3.1) implies that we can rewrite (3.4) as

$$
\mathbb{E}^{2}\left\{\bar{F}_{T}\left(S_{n+1}\right)\right\} \leq \mathbb{E}\left\{\bar{F}_{T}\left(S_{n}\right)\right\} \mathbb{E}\left\{\bar{F}_{T}\left(S_{n+2}\right)\right\} .
$$

The next proposition provides conditions under which (3.2) is verfied.

Proposition 3.1. Let $\left(X_{n}\right)_{n=1,2, \ldots}$ be an arbitrary sequence of interarrival times and $T$ a random time independent of them. Assume that $T$ is a DFR $R V$. If $\left(X_{1}, X_{2}\right)$ is associated and $X_{2} \leq \mathrm{sT} X_{1}$ then

$$
\mathbb{E}^{2}\left\{\bar{F}_{T}\left(X_{1}\right)\right\} \leq \mathbb{E}\left\{\bar{F}_{T}\left(S_{2}\right)\right\} \bar{F}_{T}(0) .
$$

Proof. Firstly, we note that, as $\bar{F}_{T}$ is a DFR survival function then the survival function $\bar{F}_{T}(\cdot) / \bar{F}_{T}(0)$ satisfies the NWU property (recall (ii) and (iii) of Remark 2.1 ). Thus,

$$
\frac{\bar{F}_{T}\left(x_{1}+x_{2}\right)}{\bar{F}_{T}(0)} \geq \frac{\bar{F}_{T}\left(x_{1}\right)}{\bar{F}_{T}(0)} \frac{\bar{F}_{T}\left(x_{2}\right)}{\bar{F}_{T}(0)}, \quad x_{1}, x_{2} \geq 0
$$

and, therefore,

$$
\bar{F}_{T}\left(x_{1}+x_{2}\right) \geq \frac{\bar{F}_{T}\left(x_{1}\right) \bar{F}_{T}\left(x_{2}\right)}{\bar{F}_{T}(0)}, \quad x_{1}, x_{2} \geq 0 .
$$

We take $x_{1}=X_{1}$ and $x_{2}=X_{2}$ to write

$$
\bar{F}_{T}\left(X_{1}+X_{2}\right) \geq \frac{\bar{F}_{T}\left(X_{1}\right) \bar{F}_{T}\left(X_{2}\right)}{\bar{F}_{T}(0)} .
$$


We take expectations in the previous inequality to obtain

$$
\mathbb{E}\left\{\bar{F}_{T}\left(S_{2}\right)\right\} \geq \frac{\mathbb{E}\left\{\bar{F}_{T}\left(X_{1}\right) \bar{F}_{T}\left(X_{2}\right)\right\}}{\bar{F}_{T}(0)} .
$$

As $\left(X_{1}, X_{2}\right)$ is associated and the functions $g_{i}: \boldsymbol{R}^{2} \rightarrow \boldsymbol{R}, i=1,2$ defined by $g_{i}\left(x_{1}, x_{2}\right)=$ $\bar{F}_{T}\left(x_{i}\right), i=1,2$ are decreasing, recalling Remark 2.3, we can write

$$
\mathbb{E}\left\{\bar{F}_{T}\left(X_{1}\right) \bar{F}_{T}\left(X_{2}\right)\right\} \geq \mathbb{E}\left\{\bar{F}_{T}\left(X_{1}\right)\right\} \mathbb{E}\left\{\bar{F}_{T}\left(X_{2}\right)\right\} .
$$

Since $X_{2} \leq_{\mathrm{ST}} X_{1}$, by Remark 2.2, we have

$$
\mathbb{E}\left\{\bar{F}_{T}\left(X_{1}\right)\right\} \mathbb{E}\left\{\bar{F}_{T}\left(X_{2}\right)\right\} \geq \mathbb{E}^{2}\left\{\bar{F}_{T}\left(X_{1}\right)\right\} .
$$

The proof follows from (3.6)-(3.8).

In the next result, we provide sufficient conditions in order to verify (3.3) when we can derive distributional versions of $\left(X_{n+1}, X_{n+2}\right)$ given $\left(X_{1}, \ldots, X_{n}\right)$ satisfying (3.2).

Proposition 3.2. Let $\{N(t): t \geq 0\}$ be a general counting process with interrenewal epochs $\left(X_{n}\right)_{n=1,2, \ldots}$. Let $T$ be a DFR random time independent of the process. Let $n$ be a fixed natural number and assume that there exists a distributional version of $\left(X_{n+1}, X_{n+2}\right)$ given $\left(X_{1}, \ldots, X_{n}\right)$ (say $\left.\left\{\left(Z_{n+1}^{x}, Z_{n+2}^{x}\right), \boldsymbol{x} \in \boldsymbol{R}_{+}^{n}\right\}\right)$ satisfying, for every DFR survival function $\bar{H}$ with $\bar{H}(0)=1$,

$$
\mathbb{E}^{2}\left\{\bar{H}\left(Z_{n+1}^{x}\right)\right\} \leq \mathbb{E}\left\{\bar{H}\left(Z_{n+1}^{x}+Z_{n+2}^{x}\right)\right\}, \quad x \in N_{n},
$$

in which $N_{n} \subseteq R_{+}^{n}$ is such that $\mathbb{P}\left\{\left(X_{1}, \ldots, X_{n}\right) \in N_{n}\right\}=1$. Then,

$$
\mathbb{E}^{2}\left\{\bar{F}_{T}\left(S_{n+1}\right)\right\} \leq \mathbb{E}\left\{\bar{F}_{T}\left(S_{n}\right)\right\} \mathbb{E}\left\{\bar{F}_{T}\left(S_{n+2}\right)\right\} .
$$

Proof. Note that, without lost of generality, we can assume that (3.9) is verified for all $\boldsymbol{x} \in \mathbb{R}_{+}^{n}$. Outside of $N_{n}$, we can always define $\left(Z_{n+1}^{x}, Z_{n+2}^{x}\right)=(0,0)$, which obviously satisfies (3.9). Thus, in order to verify inequality (3.10), consider $X:=\left(X_{1}, \ldots, X_{n}\right)$, and for each $\boldsymbol{x}:=\left(x_{1}, \ldots, x_{n}\right) \in \mathbb{R}_{+}^{n}$, denote $s_{n}:=x_{1}+\cdots+x_{n}$. As $\bar{F}_{T}$ is a DFR survival function then the survival function $\bar{H}$ defined as

$$
\bar{H}(z)=\frac{\bar{F}_{T}\left(s_{n}+z\right)}{\bar{F}_{T}\left(s_{n}\right)} \quad \text { for } z \geq 0
$$

verifies the DFR property (recall (ii) of Remark 2.1). Using (2.3), we can write

$$
\mathbb{E}\left\{\bar{F}_{T}\left(S_{n+1}\right)\right\}=\int_{\mathbb{R}_{+}^{n}} \mathbb{E}\left\{\bar{F}_{T}\left(s_{n}+Z_{n+1}^{\boldsymbol{x}}\right)\right\} \mathrm{d} F_{\boldsymbol{X}}(\boldsymbol{x})=\int_{\mathbb{R}_{+}^{n}} \bar{F}_{T}\left(s_{n}\right) \mathbb{E}\left\{\bar{H}\left(Z_{n+1}^{\boldsymbol{x}}\right)\right\} \mathrm{d} F_{\boldsymbol{X}}(\boldsymbol{x}) .
$$

As $\bar{H}(0)=1$, we use (3.9) and the Cauchy-Schwartz inequality to obtain

$$
\begin{aligned}
\int_{\mathbb{R}_{+}^{n}} \bar{F}_{T}\left(s_{n}\right) \mathbb{E}\left\{\bar{H}\left(Z_{n+1}^{x}\right)\right\} \mathrm{d} F_{\boldsymbol{X}}(\boldsymbol{x}) & \leq \int_{\mathbb{R}_{+}^{n}} \bar{F}_{T}\left(s_{n}\right) \mathbb{E}^{1 / 2}\left\{\bar{H}\left(Z_{n+1}^{\boldsymbol{x}}+Z_{n+2}^{\boldsymbol{x}}\right)\right\} \mathrm{d} F_{\boldsymbol{X}}(\boldsymbol{x}) \\
& =\int_{\mathbb{R}_{+}^{n}} \bar{F}_{T}^{1 / 2}\left(s_{n}\right) \mathbb{E}^{1 / 2}\left\{\bar{F}_{T}\left(s_{n}+Z_{n+1}^{\boldsymbol{x}}+Z_{n+2}^{\boldsymbol{x}}\right)\right\} \mathrm{d} F_{\boldsymbol{X}}(\boldsymbol{x}) \\
& \leq \mathbb{E}^{1 / 2}\left\{\bar{F}_{T}\left(S_{n}\right)\right\} \mathbb{E}^{1 / 2}\left\{\bar{F}_{T}\left(S_{n+2}\right)\right\} .
\end{aligned}
$$

Thus, from (3.12) and (3.13) we prove (3.10). 
Now, we are in a position to prove the main result of this section.

Theorem 3.1. Let $\{N(t): t \geq 0\}$ be a general counting process with interrenewal epochs $\left(X_{n}\right)_{n=1,2, \ldots}$. Let $T$ be a random time independent of the process. Assume that

(i) $T$ is a DFR $R V$,

(ii) $T$ and $X_{1}$ do not have 0 as a common discontinuity point,

(iii) $\left(X_{1}, X_{2}\right)$ is associated and $X_{2} \leq \mathrm{ST} X_{1}$.

(iv) For each $n=1,2, \ldots$ there exists a distributional version of $\left(X_{n+1}, X_{n+2}\right)$ given $\left(X_{1}, \ldots, X_{n}\right)$ (say $\left\{\left(Z_{n+1}^{x}, Z_{n+2}^{x}\right), x \in R_{+}^{n}\right\}$ satisfying, on a set $N_{n} \subseteq R_{+}^{n}$ such that $\mathbb{P}\left\{\left(X_{1}, \ldots, X_{n}\right) \in N_{n}\right\}=1$, that $\left(Z_{n+1}^{x}, Z_{n+2}^{x}\right)$ is associated and $Z_{n+2}^{x} \leq \mathrm{sT} Z_{n+1}^{x}$ for all $\boldsymbol{x} \in N_{n}$.

Then $N(T)$ is $d D F R$.

Proof. The result will follow by Lemma 3.1. Firstly, (3.2) follows by condition (iii) above and Proposition 3.1. To prove (3.3), fix $n$ and consider $\bar{H}$ a DFR survival function with $\bar{H}(0)=1$. Condition (iv) above, together with Proposition 3.1 applied to $\left(X_{1}, X_{2}\right):=$ $\left(Z_{n+1}^{x}, Z_{n+2}^{x}\right), x \in N_{n}$ implies that

$$
\mathbb{E}^{2}\left\{\bar{H}\left(Z_{n+1}^{x}\right)\right\} \leq \mathbb{E}\left\{\bar{H}\left(Z_{n+1}^{x}+Z_{n+2}^{x}\right)\right\}, \quad x \in N_{n} .
$$

This, together with Proposition 3.2, proves (3.3), and, therefore, the dDFR property for $N(T)$.

As an immediate consequence of Theorem 3.1, and taking into account that independence implies association (recall Remark 2.4), we have the following DFR preservation property for independent interarrival times.

Corollary 3.1. Let $\{N(t): t \geq 0\}$ be a general counting process with interarrival times $\left(X_{n}\right)_{n=1,2, \ldots}$. Let $T$ be a nonnegative $R V$, independent of the process. Suppose that the following assumptions hold:

(i) that $T$ is a DFR RV,

(ii) $T$ and $X_{1}$ do not have simultaneously positive mass at 0 ,

(iii) the interarrival times are independent,

(iv) $X_{n+1} \leq_{\mathrm{sT}} X_{n}, \quad n=1,2, \ldots$.

Then $N(T)$ is dDFR.

\section{Applications in queueing models}

In this section we present some applications of Corollary 3.1 in queueing models. In the first result we provide conditions for the preservation property in a pure birth process.

Corollary 4.1. Let $\{N(t): t \geq 0\}$ be a pure birth process with rates $\lambda_{n}$ increasing in $n$. If $T$ is a DFR random time independent of the process then $N(T)$ is $d D F R$. 
Proof. A pure birth process is a counting process with independent interarrival times such that the $n$th interarrival time $X_{n}$ is an exponential RV with mean $1 / \lambda_{n}, n=1,2, \ldots$. Then the result is derived from Corollary 3.1, taking into account that the RVs $X_{n}$ are decreasing in the usual stochastic order if the sequence $\lambda_{n}$ is increasing in $n$.

Remark 4.1. Corollary 4.1 implies, in particular, that a Yule process stopped at a DFR random time is dDFR. Recall that a Yule process has rates $\lambda_{n}=n \lambda$ for some fixed $\lambda>0$.

Also as a consequence of Corollary 3.1, in the following results we provide some applications concerning preservation results for queueing models. This example makes use of the distributional Little's law [12] and uses previous preservation results due to [19]. To this end, consider a queueing model which is a first in, first out (FIFO) GI/GI/1. This means that times between the arrivals of customers to the system are i.i.d. RVs, $\left(A_{n}\right)_{n=1,2, \ldots}$, and the service times $\left(B_{n}\right)_{n=1,2, \ldots}$ are i.i.d. RVs, both being independent processes. Also, there is only one server, which follows the FIFO discipline. We will assume, in addition, that $\mathbb{E}\left\{B_{1}\right\}<\mathbb{E}\left\{A_{1}\right\}$ (stability of the queue). It is well known that the steady-state number of customers waiting in a queue $L^{*}$ (the total number of customers waiting to be served in a steady-state queue) follows the distributional Little's law (see [12] for the original result, or [15, p. 217], for example, for the specific application we are going to deal with); that is, $L^{*}=\mathrm{ST} N(T)$, in which $\{N(t), t \geq 0\}$ is an equilibrium renewal process and $T$ is a random time independent of it. These quantities are defined in terms of the queue as follows.

- The renewal process $\{N(t), t \geq 0\}$ is a time-stationary version of the renewal counting process describing the arrivals of customers $\left(A_{n}\right)_{n=1,2, \ldots}$, that is, with independent interarrival times. The first interarrival time $X_{1}$ has the equilibrium distribution of $A_{1}$, given by

$$
\mathbb{P}\left\{X_{1}>t\right\}=\frac{1}{\mathbb{E}\left\{A_{1}\right\}} \int_{t}^{\infty} \mathbb{P}\left\{A_{1}>u\right\} \mathrm{d} u \quad \text { for } t \geq 0,
$$

and the following interarrival times $X_{2}, X_{3}, \ldots$ have the same distribution as $A_{1}$.

- The random time $T$ is distributed as the steady-state waiting time (the total time waiting in the queue, in the stationary situation).

On the other hand, we will use some results of Shanthikumar (cf. [19]) giving sufficient conditions for the DFR property of the steady state waiting time $T$.

We are now in a position to enunciate the following.

Corollary 4.2. Consider a FIFO GI/GI/1 queueing system, as described above. If the interarrival times of customers $\left(A_{n}\right)_{n=1,2, \ldots}$ are NWUE and the service times $\left(B_{n}\right)_{n=1,2, \ldots}$ are DFR $R V$ s then the steady-state number of customers waiting in queue $L^{*}$ is $d D F R$.

Proof. The proof is based on Corollary 3.1 and the above representation of $L^{*}$ in terms of $N(T)$, a renewal process with interarrival times $\left(X_{n}\right)_{n=1,2, \ldots}$ stopped at an independent random time $T$, as described above. First of all, the DFR property for the service times implies that $T$, the steady-state wating time, is DFR (see [19, Theorem 5.3]), and assumption (i) in Corollary 3.1 holds. To verify assumption (ii), note that $X_{1}$ has the equilibrium distribution of the arrival epochs of customers, given in (4.1). Then $X_{1}$ is continuous, and therefore $X_{1}$ and $T$ do not have 0 as a common discontinuity point in their distribution functions (despite the fact that $T$ is a compound geometric distribution, thus having strictly positive mass at the origin; see, for example, [19]). The independence assumption (iii) for the interarrival times $\left(X_{n}\right)_{n=1,2, \ldots}$ is also 
verified. As for assumption (iv), it is also known (see, for example, [14, p. 174]) that if $A_{1}$ is NWUE then its equilibrium distribution (which is the distribution of $X_{1}$ ) verifies $A_{1} \leq \mathrm{ST} X_{1}$. As noted before, the remaining interarrival times $X_{2}, X_{3}, \ldots$ are distributed as $A_{1}$ and, we have, therefore, $X_{1} \geq_{\mathrm{ST}} X_{2}={ }_{\mathrm{ST}} X_{3} \ldots$, which proves assumption (iv).

Remark 4.2. The asymptotic properties of the number $L$ of customers in a FIFO GI/GI/1 queueing system were studied in [1] (note that in Corollary 4.2, we study $L^{*}$, the number of customers in the queue). Note that if we apply Little's distributional law to $L$ (cf. [1]), $L=\mathrm{ST} N(T+B)$, where $\{N(t), t \geq 0\}$ and $T$ are as above and $B=\mathrm{ST} B_{1}$, the service time, and is independent of $\{N(t), t \geq 0\}$ and $T$. In fact, $T+B$ represent the stationary sojourn time of a customer (the time from arrival to departure from the system). Note that the conditions in Corollary 4.2 do not imply the DFR property for $L$. In fact, we can ensure the DFR property of both $T$ and $B$, but not the DFR property of $T+B$, as this property is not preserved under convolution. However, as $L^{*}=(L-1)_{+}$, the set of inequalities in Definition 2.2 for $L$ and $n=2,3, \ldots$ are verified by means of the dDFR property of $L^{*}$, but they might fail at $n=0$ or $n=1$.

A similar result to the previous one can be proved for an M/GI/1 FIFO queue. Recall that an M/GI/1 queueing system is a GI/GI/1 queueing system in which the arrival times follow an exponential distribution.

Corollary 4.3. Consider an M/GI/I FIFO queueing system. If the service times are IMRL then $L^{*}$ is $d D F R$.

Proof. In an M/GI/1 queueing system, the distribution function of the arrival times is exponential and the exponential distribution is NWUE. Then, proceeding as in the proof of Corollary 4.2, we conclude that $L^{*}=N(T)$ satisfies conditions (iii) and (iv) of Corollary 3.1. Condition (i), that is, the DFR property for $T$, is a consequence of the IMRL property for the service times (see [19, Theorem 5.2]).

Remark 4.3. Recall that the DFR property is stronger than the IMRL and NWUE properties. Therefore, Corollary 4.2 is in particular verified if both the interarrival times and the service times are DFR RVs. Also, when we consider an M/GI/1 queueing system in Corollary 4.3, we can weaken the DFR condition of the service time to the IMRL condition.

\section{Examples with dependent interarrival times.}

In this section we show with some examples that Theorem 3.1 can be applied to obtain the DFR property for counting processes with dependent interarrival times. Firstly, the following lemma will be useful in order to verify the association condition.

Lemma 5.1. Let $\left(Z_{1}, Z_{2}\right)$ be a nonnegative random vector with independent components. Take two increasing (decreasing) functions $g_{i}: \boldsymbol{R}_{+}^{2} \rightarrow \boldsymbol{R}, i=1,2$. It holds that the random vector $\left(g_{1}\left(Z_{1}, Z_{2}\right), g_{2}\left(Z_{1}, Z_{2}\right)\right)$ is associated.

Proof. We have, by the independence assumption, that $\left(Z_{1}, Z_{2}\right)$ is associated. (Recall property (ii) in Remark 2.4). On the other hand, if $g_{i}: \boldsymbol{R}_{+}^{2} \rightarrow \boldsymbol{R}$ are increasing (decreasing), we can extend them to functions $g_{i}^{*}: \boldsymbol{R}^{2} \rightarrow \boldsymbol{R}$ which are also increasing (decreasing), by putting $g_{i}^{*}\left(z_{1}, z_{2}\right)=g_{i}\left(z_{1}^{+}, z_{2}^{+}\right)$, where for any real number $z, z^{+}=\max (z, 0)$. By property (i) in Remark 2.4, $\left(g_{1}^{*}\left(Z_{1}, Z_{2}\right), g_{2}^{*}\left(Z_{1}, Z_{2}\right)\right)$ is associated and the conclusion follows as $\left(Z_{1}, Z_{2}\right)$ is nonnegative. 
Making use of previous Lemma, we present two examples of counting processes verifying the hypothesis in Theorem 3.1.

Corollary 5.1. Let $\{N(t): t \geq 0\}$ be a general counting process with interarrival times defined as

$$
X_{n}=\prod_{i=1}^{n} Y_{i} \quad \text { for } n=1,2, \ldots,
$$

where $\left(Y_{n}\right)_{n=1,2, \ldots}$ is a sequence of independent nonnegative $R V s$ such that each $Y_{i}$ has support on $[0,1]$. If $T$ is a DFR RV independent of the process and $T$ and $Y_{1}$ do not have 0 as a common discontinuity point, then $N(T)$ is $d D F R$.

Proof. Conditions (i) and (ii) in Theorem 3.1 are satisfied by assumption. For the remaining conditions, let $n=0,1,2, \ldots$. We see by Lemma 5.1 that $\left(c_{n} Y_{n+1}, c_{n} Y_{n+1} Y_{n+2}\right), c_{n} \geq 0$ is associated. In particular, for $n=0,\left(X_{1}=Y_{1}, X_{2}=Y_{1} Y_{2}\right)$ is associated. Since $Y_{1} Y_{2} \leq \mathrm{sT} Y_{1}$, by the assumption about the support of the $Y_{i}$, condition (iii) in Theorem 3.1 is satisfied. Consider now $n=1,2, \ldots$ and let $c_{n}(\boldsymbol{x})=\prod_{i=1}^{n} x_{i}, \boldsymbol{x} \in R_{+}^{n}$. Clearly, a distributional version of $\left(X_{n+1}, X_{n+2}\right)$ given $\left(X_{1}, \ldots, X_{n}\right)$ is provided by

$$
\left\{\left(Z_{n+1}^{\boldsymbol{x}}=c_{n}(\boldsymbol{x}) Y_{n+1}, Z_{n+2}^{\boldsymbol{x}}=c_{n}(\boldsymbol{x}) Y_{n+1} Y_{n+2}\right), \boldsymbol{x} \in R_{+}^{n}\right\} .
$$

A similar argument as in the case $n=0$, shows that $\left(Z_{n+1}^{x}, Z_{n+2}^{x}\right)$ verifies condition (iv) in Theorem 3.1.

Corollary 5.2. Let $\{N(t): t \geq 0\}$ be a general counting process and let $\left(Y_{n}\right)_{n=1,2, \ldots}$ be a sequence of independent nonnegative RVs. Define the interarrival times by

$$
X_{n}=\frac{1}{z+Y_{1}+\ldots+Y_{n}} \text { for } n=1,2, \ldots, z \geq 0 .
$$

Let $T$ be a DFR RV independent of the process. Then

(i) if $z>0, N(T)$ is $d D F R$,

(ii) if $\mathbb{P}\left\{Y_{1}>0\right\}=1$ and $z=0$ then $N(T)$ is $d D F R$.

Proof. Firstly, condition (ii) in Theorem 3.1 is satisfied by assumption, as $X_{1}=\left(z+Y_{1}\right)^{-1}$ has no discontinuity at the origin. Second, for each $n=0,1,2, \ldots$, we see by Lemma 5.1 that under the assumptions in parts (i) and (ii),

$$
\left(\frac{1}{c_{n}+Y_{n+1}}, \frac{1}{c_{n}+Y_{n+1}+Y_{n+2}}\right) \text { for } c_{n}>0,
$$

is associated and, obviously,

$$
\frac{1}{c_{n}+Y_{n+1}} \geq \mathrm{sT} \frac{1}{c_{n}+Y_{n+1}+Y_{n+2}} \text { for } c_{n}>0 .
$$

Thus, for (i), we conclude from (5.2) that the following vector is associated:

$$
\left(X_{1}=\frac{1}{z+Y_{1}}, X_{2}=\frac{1}{z+Y_{1}+Y_{2}}\right) .
$$


Moreover, $X_{1} \geq_{\mathrm{ST}} X_{2}$ by (5.3). For part (ii) of Lemma 5.1, $\left(X_{1}=Y_{1}^{-1}, X_{2}=\left(Y_{1}+Y_{2}\right)^{-1}\right)$ is also associated using property (iii) in Remark 2.4, as the random vector in (5.4) converges in distribution to $\left(Y_{1}^{-1},\left(Y_{1}+Y_{2}\right)^{-1}\right)$, as $z \downarrow 0$ (in fact, the convergence is almost sure). As in this case we trivially have $X_{1} \geq_{\mathrm{ST}} X_{2}$, condition (iii) in Theorem 3.1 is satisfied in both cases. For condition (iv), observe that, using (5.1), we have

$$
\left(X_{n+1}=\frac{1}{X_{n}^{-1}+Y_{n+1}}, X_{n+2}=\frac{1}{X_{n}^{-1}+Y_{n+1}+Y_{n+2}}\right) \text { for } n=1,2, \ldots
$$

We also see by (5.1) that $\mathbb{P}\left\{X_{n}>0\right\}=1$, in both cases (i) and (ii). Thus, if we take $N_{n}=$ $\left\{\boldsymbol{x} \in R_{+}^{n} \mid x_{n}>0\right\}$, we have $\mathbb{P}\left\{\left(X_{1}, \ldots, X_{n}\right) \in N_{n}\right\}=1$. Then a distributional version of $\left(X_{n+1}, X_{n+2}\right)$ given $\left(X_{1}, \ldots, X_{n}\right)$ can be defined by taking

$$
\left(Z_{n+1}^{x}=\frac{1}{x_{n}^{-1}+Y_{n+1}}, Z_{n+2}^{x}=\frac{1}{x_{n}^{-1}+Y_{n+1}+Y_{n+2}}\right), \quad x \in N_{n} .
$$

Thus, the conditions are satisfied in order to apply (5.2) and (5.3) to conclude that $\left(Z_{n+1}^{x}\right.$, $Z_{n+2}^{x}$ ), $\boldsymbol{x} \in N_{n}$ verifies condition (iv) in Theorem 3.1.

\section{Acknowledgements}

The authors would like to thank the anonymous referee for their valuable comments and careful reading of earlier versions of this manuscript.

This work has been supported by the Spanish Ministerio de Educación y Ciencia Research Projects MTM2010-15311 and MTM2012-36603-C02-02, and by the European Regional Development Fund (FEDER). The first and second authors acknowledge the support of Diputación General de Aragón (DGA) S11 and E64, respectively.

\section{References}

[1] Asmussen, S., Klüppelberg, C. And Sigman, K. (1999). Sampling at subexponential times, with queueing applications. Stoch. Process. Appl. 79, 265-286.

[2] BADía, F. G. (2011). Hazard rate properties of a general counting process stopped at an independent random time J. Appl. Prob. 48, 56-67.

[3] Badía, F. G. And SAlehi, E. T. (2012). Preservation of reliability classes associated with the mean residual life by a renewal process stopped at a random time Appl. Stoch. Models Business Industry. 28, 381-394.

[4] Badía, F. G. And SANGÜESA, C. (2008). Preservation of reliability classes under mixtures of renewal processes. Prob. Eng. Inf. Sci. 22, 1-17.

[5] Barlow, R. E. ANd Proschan, F. (1975). Statistical Theory of Reliability and Life Testing. Holt, Reinhart and Winston, New York.

[6] Cox, D. R. (1962). Renewal Theory. Methuen, London.

[7] Esary, J. D., Marshall, A. W. and Proschan, F. (1973). Shock models and wear processes. Ann. Prob. 1, 627-649.

[8] Esary, J. D., Proschan, F. and Walkup, D. W. (1967). Association of random variables, with applications. Ann. Math. Statist. 38, 1466-1474.

[9] Finkelstein, M. S. and Esaulova, V. (2001). Why the mixture failure rate decreases. Reliab. Eng. Syst. Safe. 71, 173-177.

[10] Grandell, J. (1997). Mixed Poisson Processes. Chapman \& Hall, London.

[11] Gurland, J. and Sethuraman, J. (1995). How pooling failure data may reverse increasing failure rates. J. Amer. Statist. Assoc. 90, 1416-1423.

[12] Haji, R. ANd Newell, G. F. (1971). A relation between stationary queue and waiting time distributions. J. Appl. Prob. 8, 617-620.

[13] Kallenberg, O. (2002). Foundations of Modern Probability, 2nd Edn. Springer, New York.

[14] Marshall, A. W. and Olkin, I. (2007). Life Distributions. Springer, New York. 
[15] Müller, A. And Stoyan, D. (2002). Comparison Methods for Stochastic Models and Risks. John Wiley, Chichester.

[16] Proschan, F. (1963). Theoretical explanation of observed decreasing failure rate. Technometrics 5, 375-383.

[17] Ross, S. M., Shanthikumar, J. G. And Zhu, Z. (2005). On increasing-failure-rate random variables. J. Appl. Prob. 42, 797-809.

[18] Shaked, M. and Shanthikumar, J. G. (1988). Stochastic convexity and its applications. Adv. Appl. Prob. 20, 427-446.

[19] Shanthikumar, J. G. (1988). DFR property of first-passage times and its preservation under geometric compounding. Ann. Prob. 16, 397-406.

[20] Song, R., Karon, J. M., White, E. and Goldbaum, G. (2006). Estimating the distribution of a renewal process from times at which events from an independent process are detected. Biometrics 62, 838-846. 